\title{
Noncommutative Geometry Modified Non-Gaussianities of Cosmological Perturbation
}

\author{
Kejie Fang ${ }^{1}$, Bin Chen $^{1,2}$, Wei Xue ${ }^{1}$ \\ ${ }^{1}$ Department of Physics, Peking University, Beijing 100871, P.R.China \\ 2 the Abdus Salam International Center for Theoretical Physics, \\ Strada Costiera 11, 34014 Trieste, Italy
}

\begin{abstract}
We investigate the noncommutative effect on the non-Gaussianities of primordial cosmological perturbation. In the lowest order of string length and slow-roll parameter, we find that in the models with small speed of sound the noncommutative modifications could be observable if assuming a relatively low string scale. In particular, the dominant modification of non-Gaussianity estimator $f_{N L}$ could reach $O(1)$ in DBI inflation and K-inflation. The corrections are sensitive to the speed of sound and the choice of string length scale. Moreover the shapes of the corrected non-Gaussianities are distinct from that of ordinary ones.
\end{abstract}

\section{Introduction}

Inflation[1] is a very successful paradigm of the very early universe. It can naturally solve several very tough cosmological problems without fine tuning. Furthermore, it predicts a nearly scale invariant Gaussian CMB spectrum, which has been confirmed in the experiments [2]. However, one of the problems with inflation is that there are too many inflationary models. It is necessary to find the signatures which could distinct various models. With the development of precise cosmology, we expect new experiments to constrain a large amount of inflation models and to make the paradigm 
more clear. The scalar spectral index and its running, the gravitational wave and nonGaussian component of the primordial fluctuations are among the important probes to detect different inflation models. In this paper, we mainly discuss non-Gaussianity.

In recent years, the non-Gaussianities of primordial perturbation opens another window other than power spectrum to study different scenarios of inflation models. After systematic analysis [3, 4, 5, 6], it is found that non-Gaussianity estimator $f_{N L}$ is of order $O(\epsilon)$ in the ordinary "slow-roll" inflation and thus is unobservable. In some particular models with non-trivial dynamics, such as ghost inflation[10] and those with sufficiently small speed of sound, including DBI model[11, 12] and K-inflation [20, 21], the unsuppressed non-Gaussianity is potentially detectable in future experiments[7, 12. Furthermore, since the shape of non-Gaussianity is more multiple than that of power spectrum, it could be used to distinguish different inflation models through the classification of those configuration of $k$ modes that determine the maximum of three-point function [8].

Non-Gaussianity could also be used to study various trans-Planckian physics proposals. Deviation from the standard Bunch-Davies vacuum was considered in [7, 12] where the correction is found to be $O\left(\frac{H}{\Lambda}\right)$ where $\Lambda$ is the energy scale on which the modes are generated. In [9], higher dimensional operators were introduced in the inflaton Lagrangian. This modification could enhance the non-Gaussian effect if the energy cutoff is not too high; but it is difficult to exceed $f_{N L} \sim 1$. In this paper, we consider another trans-Planckian scenario which is based on noncommutative geometry.

In string theory, a promising candidate of quantum gravity, one may use perturbative string or nonperturbative object D-brane to probe the spacetime geometry. Due to the extensive nature of the string, or stringy effect, or strong string interaction, the picture of spacetime geometry in string theory could be very different from the usual one. Especially, very near the cosmological singularity, the usual concept of commutative geometry may break down completely. A better description could be noncommutative geometry[22], in terms of the algebra generated by noncommutative coordinates

$$
\left[x^{\mu}, x^{\nu}\right]=i \theta^{\mu \nu}
$$

One natural way to get noncommutative geometry is to consider the D-brane in the 
presence of constant NS-NS magnetic B field[23]. In this case, the spatial coordinates are noncommutative

$$
\left[x^{i}, x^{j}\right]=i \theta^{i j}
$$

where $\theta^{i j}$ depends on the background flux, while space-time is commutative. Similarly one can obtain space-time noncommutativity by placing D brane in constant electric field, but the theory is no more unitary in this case [24, 25, 26, 27, 28].

Another way to realize the noncommutative relation among coordinates, especially between space and time, is to start from the stringy uncertainty relation

$$
\Delta x_{p} \Delta t \geq l_{s}^{2}
$$

Equivalently one may assume $\left[t, x_{p}\right]=i l_{s}^{2}$. This kind of noncommutative relation have been applied to the study of inflationary universe [30, 31, 32]. In [30], Brandenberger and Ho started from (1.3) and discussed the cosmological implications of such relation. To keep the background isometry intact, they actually considered the noncommutative relation between radial coordinate $r$ and time $t$, however, as we mentioned before, the space-time noncommutative relation may violate the unitarity of the theory.

In this paper, in order to keep unitarity, we choose to set the space-time components $\theta^{0 i}$ zero. The cosmological imprint based on this kind of noncommutativity has been studied by many authors [34, 35, 36, 37, 38]. Meanwhile, without losing generality, we choose a particular frame in which the only non-vanishing space-space component of $\theta^{\mu \nu}$ in comoving coordinate is

$$
\left[x^{1}, x^{2}\right]=i \theta^{12}
$$

We find the calculation is much more involved if one chooses to keep all possible spatial noncommutativity but this won't change the main result we obtained from this particular frame. We also require that noncommutativity following from (1.4) only dominates at small length scale. A simple form of $\theta^{12}$ realizes this condition is 38$]$

$$
\theta^{12}=\frac{l_{s}^{2}}{a^{2}}
$$

where $l_{s}$ is the string length. In this case, the noncommutativity in physical coordinate remains to be a constant, $\left[x_{p}^{1}, x_{p}^{2}\right]=i l_{s}^{2}$, and the noncommutativity of comoving 
coordinate is diluted by the expansion of universe. However, with this choice, the isotropy of the spacetime is now broken. Nevertheless, if the noncommutativity is very small, the breaking of isotropy could be ignorable but the physical implication could be observable. In this paper, we will focus on this case.

In most of the inflation models, the non-Gaussianity is quite small and hard to be detected. The noncommutative corrections of non-Gaussianity is even smaller. This drives us to work on the models with big non-Gaussianity. We will discuss the noncommutative effect in string-inspired DBI inflation model and k-inflation model. We find that in both models the noncommutative modification of non-Gaussianity estimator can reach $O(1)$ or even larger with a small speed of sound and a relatively low string scale. We also determine the shape of corrected non-Gaussianity, which is different from the usual ones. Though the non-Gaussianity estimator could be overshadowed, the shape is a distinctive signature to be probed by the future experiments. On the contrary, future experiments could set bound of the noncommutativity and be used for crosschecking along with other experiments, like atomic experiments.

The paper is organized as follows. In section 2, we give a brief review of the ADM formalism to discuss the perturbations in a general inflation lagrangian. In section 3, we study the noncommutative modifications to general inflation models. In section 4, we focus on two inflation models with observable non-Gaussianity. In section 5, we end with conclusion and discussions.

\section{Perturbations in general inflation models}

Let us start with a general Lagrangian $P(X, \phi)$ which can be used to describe a broad class of inflation models. The action is of the form as follows,

$$
S=\frac{1}{2} \int d^{4} x \sqrt{-g}[R+2 P(X, \phi)]
$$

where $\phi$ is the inflaton field and $X=-\frac{1}{2} g^{\mu \nu} \partial_{\mu} \phi \partial_{\nu} \phi$. We set Planck mass $M_{p l}=$ $(8 \pi G)^{-\frac{1}{2}}=1$ and adopt the metric signature $(-1,1,1,1)$.

In order to proceed, it is convenient to work in the ADM metric formalism,

$$
d^{2} s=-N^{2} d^{2} t+h_{i j}\left(d x^{i}+N^{i} d t\right)\left(d x^{j}+N^{j} d t\right)
$$


where $h_{i j}=a^{2}(1+2 \zeta) \delta_{i j}$, with $a$ the scale factor which grows quasi-exponentially during inflation and $\zeta$ the scalar perturbation of metric. In this paper, we do not consider the tensor perturbation. Since in the ADM formalism $N$ and $N^{i}$ are Lagrangian multipliers, the metric in terms of $\zeta$ can be determined by solving the constraint equations of $N$ and $N^{i}$ in a given gauge, rather by solving the Einstein equation, which makes the calculation relatively simpler.

There are two main gauges in which we can calculate the perturbation action. One gauge is the comoving gauge, that is $\delta \phi=0$. In this gauge, $\zeta$ is the curvature scalar on a comoving hypersurface and it directly seeds on the later generation of large scale structure and anisotropy of microwave background radiation. It is straightforward to find out that $\zeta$ remains constant after horizon exit. Although the physical meaning is manifest within this gauge, it is rather complicated to analyze the order of the perturbation action with respect to the slow-roll parameters, which is important in determining the magnitude of correlation functions. In this gauge, the third order action of perturbation is apparently of order $O\left(\epsilon^{0}\right)$, where $\epsilon$ represents the slow-roll parameter; however after doing a lot of integration by part the action is actually of order $O\left(\epsilon^{2}\right)$ [3, 7]. The other gauge with $\zeta=0$ is called uniform density gauge. The gauge transformation linking the two gauge is $\zeta=\frac{H}{\phi} \delta \phi$, where $H$ is the Hubble parameter $H=\frac{\dot{a}}{a}$. One could easily recover the exact order $O\left(\epsilon^{2}\right)$ of the action by doing a gauge transformation of the perturbation action of $\delta \phi[3]$. In order to calculate the correlation function of metric perturbation in this gauge, we first calculate the correlation function of $\delta \phi$ and then transform it to $\zeta$ just after horizon exit, which remains constant ever since.

Below we adopt the uniform density gauge $\zeta=0$ to carry out the calculation. Substituting the ADM metric into the action(2.1), we get

$$
S=\frac{1}{2} \int d t d^{3} x \sqrt{h} N\left(R^{(3)}+2 P\right)+\frac{1}{2} \int d t d^{3} x \sqrt{h} N^{-1}\left(E_{i j} E^{i j}-E^{2}\right),
$$

where $h=\operatorname{det}\left(h_{i j}\right), E_{i j}=\frac{1}{2}\left(\dot{h}_{i j}-\nabla_{i} N_{j}-\nabla_{j} N_{i}\right), E=E_{i}^{i} . R^{(3)}$ is the Ricci scalar calculated in the three-dimensional hypersurface with metric $h_{i j}$, and $\nabla_{i}$ is the covariant differential coefficient defined on the hypersurface. Since $N$ and $N^{i}$ are Lagrangian multipliers, we can obtain two constraint equations from them, which are

$$
R^{(3)}+2 P-2 N^{-2} P_{, X}\left(\dot{\phi}^{2}-2 N^{i} \dot{\phi} \partial_{i} \phi+N^{i} N^{i} \partial_{i} \phi \partial_{j} \phi\right)-N^{-2}\left(E_{i j} E^{i j}-E^{2}\right)=0,
$$




$$
-N P_{, X}\left(2 N^{-2} \dot{\phi} \partial \phi-\frac{2 N^{j}}{N^{2}} \partial_{i} \phi \partial_{j} \phi\right)+2\left(\nabla_{j}\left(N^{-1} E_{i}^{j}\right)-\nabla_{i}\left(N^{-1} E\right)\right)=0 .
$$

We divide $\phi$ into the isotropic background $\phi_{0}(t)$ and the fluctuation $\varphi, \phi=\phi_{0}+\varphi$. In order to evaluate the third order action of perturbation, we only need to solve the equations of $N$ and $N^{i}$ to the first order of $\varphi$, since the second and third order of solutions, when substituted into the action, will be multiplied with the first and zeroth order Hamiltonian constraint of the action respectively and thus vanish. In fact, to calculate the $n$-th order action of perturbation, one only needs the solutions of $N$ and $N^{i}$ to $(n-2)$-th order [7]. Following [3], we decompose $N^{i}$ into two parts $N_{i}=\tilde{N}_{i}+\partial_{i} \psi$, where $\partial_{i} \tilde{N}^{i}=0$ and $N_{i}$ is lowered by $h_{i j}$ through $N^{i}$. Then we expand them to the first order of $\varphi$,

$$
N=1+\alpha_{1}, \quad \tilde{N}_{i}=N_{i}^{(1)}, \quad \psi=\psi_{1},
$$

where $\alpha_{1}, N_{i}^{(1)}$ and $\psi_{1}$ are of order $O(\varphi)$. Substituting (2.6) into (2.4) and (2.5), and solving them to $O(\varphi)$, we obtain the solution,

$$
\begin{aligned}
& \alpha_{1}=\frac{P_{, X_{0}} \dot{\phi}_{0}}{2 H} \varphi, \quad N_{i}^{(1)}=0, \\
& \partial^{2} \psi_{1}=\frac{1}{4 H}\left(\left(2 P_{, \phi_{0}}+\dot{\phi}_{0}^{3} H^{-1} P_{, X_{0}}^{2}-2 P_{, X_{0} \phi_{0}} \dot{\phi}_{0}^{2}+\dot{\phi}_{0}^{5} H^{-1} P_{, X_{0}} P_{, X_{0} X_{0}} \varphi\right.\right. \\
& \left.\left.-6 H P_{, X_{0}} \dot{\phi}_{0}\right) \varphi-\left(2 P_{, X_{0}} \dot{\phi}_{0}+2 P_{, X_{0} X_{0}} \dot{\phi}_{0}\right) \dot{\varphi}\right) \text {, }
\end{aligned}
$$

where the subindex 0 represents the background value.

It will be more succinct to express the solutions using the slow-roll parameter $\epsilon$ and the "speed of sound" $c_{s}$,

$$
\begin{gathered}
\epsilon=-\frac{\dot{H}}{H^{2}}=\frac{X_{0} P_{X_{0}}}{H^{2} M_{p l}^{2}}, \\
c_{s}^{2}=\frac{d P}{d E}=\frac{P_{X_{0}}}{P_{, X_{0}}+2 X_{0} P_{,_{0} X_{0}}},
\end{gathered}
$$

where $E=2 X P_{, X}-P$ is the energy of inflaton field. Keeping with the lowest order of slow-roll parameter, equations (2.7) (2.8) can be written as

$$
\begin{gathered}
\alpha_{1}=\frac{H \epsilon}{\dot{\phi}_{0}} \varphi \\
\partial^{2} \psi_{1}=\left(\frac{P_{\phi_{0}}}{2 H}-\frac{3 H^{2}}{\dot{\phi}_{0}} \epsilon\right) \varphi-\frac{H \epsilon}{\dot{\phi}_{0} c_{s}^{2}} \dot{\varphi}
\end{gathered}
$$


We omit the subindex 1 in these expressions in the following section for simplicity.

Substituting (2.11) and (2.12) into (2.3) and expanding it to the second order of perturbation, we attain the free field action of fluctuation

$$
S_{2}=\frac{1}{2} \int d^{4} x a^{3}\left(\frac{P_{, X_{0}}}{c_{s}^{2}} \dot{\varphi}^{2}-P_{, X_{0}}(\partial \varphi)^{2}\right) .
$$

The equation of motion is

$$
(a \varphi)^{\prime \prime}+\left(c_{s}^{2} k^{2}-\frac{a^{\prime \prime}}{a}\right) a \varphi=0
$$

where the prime denotes derivative with respect to conformal time and we have assumed $P_{, X_{0}}$ and $c_{s}$ to be time independent. The classical solution is

$$
\varphi=\frac{\dot{\phi}_{0}}{\sqrt{4 \epsilon c_{s} k^{3}}}\left(1+i c_{s} k \eta\right) e^{-i c_{s} k \eta}
$$

where we choose the standard Bunch-Davies vacuum to fix the coefficient. With the same procedure one could obtain the third order action of perturbation which has been studied thoroughly in [7] in the comoving gauge.

\section{Noncommutative modification}

In the noncommutative spacetime, the functions are better described by the operators in Hilbert space. This is very similar to the case in quantum mechanics, where one has a noncommutative phase space. The product of functions may be taken as the multiplication of the operators. But an efficient way to define product is by so-called Moyal product, whose expansion in curved spacetime gives 38.

$$
f \star g \equiv \sum_{k=0}^{\infty} \frac{1}{k !}\left(\frac{i}{2}\right)^{k} \theta^{\mu_{1} \nu_{1}} \cdots \theta^{\mu_{k} \nu_{k}}\left(D_{\mu_{1}} \cdots D_{\mu_{k}} f\right)\left(D_{\nu_{1}} \cdots D_{\nu_{k}} g\right),
$$

where $D_{\mu}$ is the covariant derivative coefficient in the curved spacetime.

The star product could be extended to multiple function situation, at quadratic order in $\theta^{\mu \nu}$,

$$
f_{1} \star \cdots \star f_{n}=\left(1+\frac{i}{2} \theta^{\mu \nu} \sum_{a<b} D_{\mu}^{a} D_{\nu}^{b}-\frac{1}{8} \theta^{\mu \nu} \theta^{\rho \sigma} \sum_{a<b, c<d} D_{\mu}^{a} D_{\nu}^{b} D_{\rho}^{c} D_{\sigma}^{d}\right) f_{1} \cdots f_{n} .
$$

To incorporate the noncommutative effect, we replace the ordinary product in the inflaton Lagrangian $P(X, \phi)$ with the star product. Suppose that $P(X, \phi)$ relies 
on $\phi$ through function $V(\phi) 1$, which could be the inflaton potential or the warping factor in DBI model. Without losing generality, we simply consider $V(\phi)$ of the form $V(\phi)=\phi^{n}, n \geq 1$, since one could always do Taylor's expansion of a generic function. When apply the star product in the generic Lagrangian, noncommutativity changes the forms of dynamic term $X=-\frac{1}{2} \partial_{\mu} \phi \partial^{\mu} \phi$ and scalar function $V(\phi)$ as follows

$$
\begin{aligned}
X= & -\frac{1}{2}\left(\partial_{\mu} \phi\right) \star\left(\partial^{\mu} \phi\right) \\
= & -\frac{1}{2}\left(\partial_{\mu} \phi\right)\left(\partial^{\mu} \phi\right)+\frac{1}{8}\left(\theta^{12}\right)^{2} g^{\mu \nu}\left(D_{1} D_{1} D_{\mu} \phi D_{2} D_{2} D_{\nu} \phi\right. \\
& \left.-D_{1} D_{2} D_{\mu} \phi D_{2} D_{1} D_{\nu} \phi\right)+O\left(\theta^{3}\right),
\end{aligned}
$$

and

$$
\begin{aligned}
V= & \phi \star \cdots \star \phi \\
= & \phi^{n}-\frac{n(n-1)}{8}\left(\theta^{12}\right)^{2} \phi^{n-2}\left(D_{1} D_{1} \phi D_{2} D_{2} \phi-D_{1} D_{2} \phi D_{2} D_{1} \phi\right) \\
& -\frac{n(n-1)(n-2)}{24}\left(\theta^{12}\right)^{2} \phi^{n-3}\left(D_{1} D_{1} \phi D_{2} \phi D_{2} \phi+D_{2} D_{2} \phi D_{1} \phi D_{1} \phi\right. \\
& \left.-D_{1} D_{2} \phi D_{1} \phi D_{2} \phi-D_{2} D_{1} \phi D_{2} \phi D_{1} \phi\right) \\
& +O\left(\theta^{3}\right) .
\end{aligned}
$$

We find that the lowest order noncommutative modification term is of order $O\left(\theta^{2}\right)$. We only consider the correction of the lowest order $\theta^{2}$ in this paper, and denote them as $\delta_{\theta} X$ and $\delta_{\theta} V$ respectively,

$$
\begin{aligned}
\delta_{\theta} X= & \frac{1}{8}\left(\theta^{12}\right)^{2} g^{\mu \nu}\left(D_{1} D_{1} D_{\mu} \phi D_{2} D_{2} D_{\nu} \phi-D_{1} D_{2} D_{\mu} \phi D_{2} D_{1} D_{\nu} \phi\right), \\
\delta_{\theta} V= & -\frac{n(n-1)}{8}\left(\theta^{12}\right)^{2} \phi^{n-2}\left(D_{1} D_{1} \phi D_{2} D_{2} \phi-D_{1} D_{2} \phi D_{2} D_{1} \phi\right) \\
& -\frac{n(n-1)(n-2)}{24}\left(\theta^{12}\right)^{2} \phi^{n-3}\left(D_{1} D_{1} \phi D_{2} \phi D_{2} \phi+D_{2} D_{2} \phi D_{1} \phi D_{1} \phi\right. \\
& \left.-D_{1} D_{2} \phi D_{1} \phi D_{2} \phi-D_{2} D_{1} \phi D_{2} \phi D_{1} \phi\right) .
\end{aligned}
$$

One should keep in mind that all the covariant derivatives are calculated within the ADM formalism, which makes the evaluation much more involved. In some other papers in calculating large unsuppressed non-Gaussianity [10, 11, 9], because of the

\footnotetext{
${ }^{1}$ If there are more than one such functions, one just needs to include all the corrections from each function in (3.14) .
} 
particularity of the actions (they could themselves, by doing serial expansion, generate cubic terms of perturbation without involving metric corrections, i.e. $\alpha, \psi)$, the authors ignore the correlation between metric correction and inflaton perturbation in the uniform density gauge, which is subleading in slow-roll parameter, and do the calculation with isotropic FRW metric. Our calculation turns out to be the same situation when considering the noncommutativity correction.

The change of the inflation action is

$$
\delta_{\theta} S=\int d^{4} x \sqrt{-g}\left(P_{, X} \delta_{\theta} X+P_{, V} \delta_{\theta} V\right) .
$$

To obtain the third order action of perturbation, one needs to serially expand $P_{, X}$ and $P_{, V}$ around the background value and multiply with the corresponding terms in $\delta_{\theta} X$ and $\delta_{\theta} V$ which generate overall cubic terms of perturbation.

To simplify the calculation, we need to pick out the terms of leading order of slow-roll parameter. We find in this case, the leading order is $O(\epsilon)$ for $\zeta$ after gauge transformation. We decompose $\delta_{\theta} X$ into terms of different order of perturbation,

$$
\delta_{\theta} X=\left(\delta_{\theta} X\right)_{3}+\left(\delta_{\theta} X\right)_{2}+\left(\delta_{\theta} X\right)_{1}+\left(\delta_{\theta} X\right)_{0}
$$

where $\left(\delta_{\theta} X\right)_{3}$ represents the cubic terms, etc., and we do not consider the higher order of perturbation since we are only going to calculate the three-point function. The terms of lowest order of slow-roll parameter in $\left(\delta_{\theta} X\right)_{3}$ are those composed of product of two inflaton perturbation $\varphi$ and one metric correction, i.e. $\alpha$ and $\psi$. However, we do not need to take them into account to obtain the final third order action of perturbation by the reason that they will generate terms of second order in slowroll parameter after doing the gauge transformation to $\zeta$, which turns out to be the subleading terms in our calculation. As for $\left(\delta_{\theta} X\right)_{0}$ which is composed of terms of $\dot{\phi}_{0}$, it also results in subleading order terms when multiplied with the serially expanding terms of $P_{, X}$. In short, we only need $\left(\delta_{\theta} X\right)_{2}$ and $\left(\delta_{\theta} X\right)_{1}$.

The terms of lowest order of slow-roll parameter in $\left(\delta_{\theta} X\right)_{2}$ are the products of two 
inflaton perturbation, which are summarized as follows,

$$
\begin{aligned}
\left(\delta_{\theta} X\right)_{2}= & \frac{1}{8}\left(\theta^{12}\right)^{2}\left(\frac{1}{a^{2}} \partial_{1}^{2} \partial_{i} \varphi \partial_{2}^{2} \partial_{i} \varphi-\frac{1}{a^{2}} \partial_{1} \partial_{2} \partial_{i} \varphi \partial_{1} \partial_{2} \partial_{i} \varphi-H \partial_{1}^{2} \partial_{i} \varphi \partial_{i} \dot{\varphi}\right. \\
& -H \partial_{2}^{2} \partial_{i} \varphi \partial_{i} \dot{\varphi}+H^{2} \partial_{1}^{2} \partial_{i} \varphi \partial_{i} \varphi+H^{2} \partial_{2}^{2} \partial_{i} \varphi \partial_{i} \varphi+\dot{a}^{2} \partial_{1} \dot{\varphi} \partial_{1} \dot{\varphi} \\
& +\dot{a}^{2} \partial_{2} \dot{\varphi} \partial_{2} \dot{\varphi}+\dot{a}^{2} \partial_{i} \dot{\varphi} \partial_{i} \dot{\varphi}-2 H \dot{a}^{2} \partial_{1} \dot{\varphi} \partial_{1} \varphi-2 H \dot{a}^{2} \partial_{2} \dot{\varphi} \partial_{2} \varphi \\
& -2 H \dot{a}^{2} \partial_{i} \dot{\varphi} \partial_{i} \varphi+(H \dot{a})^{2} \partial_{1} \varphi \partial_{1} \varphi+(H \dot{a})^{2} \partial_{2} \varphi \partial_{2} \varphi \\
& +(H \dot{a})^{2} \partial_{i} \varphi \partial_{i} \varphi-\partial_{1}^{2} \dot{\varphi} \partial_{2}^{2} \dot{\varphi}+\partial_{1} \partial_{2} \dot{\varphi} \partial_{1} \partial_{2} \dot{\varphi}-(H a \dot{a})^{2} \dot{\varphi}^{2} \\
& -H a \dot{a} \dot{\varphi} \partial_{1}^{2} \dot{\varphi}+H a \dot{a} \dot{\varphi} \partial_{2}^{2} \dot{\varphi}+2 H \partial_{1}^{2} \dot{\varphi} \partial_{2}^{2} \varphi+2 H \partial_{1}^{2} \varphi \partial_{2}^{2} \dot{\varphi} \\
& +2 H^{2} a \dot{a} \dot{\varphi} \partial_{1}^{2} \varphi+2 H^{2} a \dot{a} \dot{\varphi} \partial_{2}^{2} \varphi-4 H \partial_{1} \partial_{2} \dot{\varphi} \partial_{1} \partial_{2} \varphi \\
& -4 H^{2} \partial_{1}^{2} \varphi \partial_{2}^{2} \varphi+4 H^{2} \partial_{1} \partial_{2} \varphi \partial_{1} \partial_{2} \varphi+a \dot{a} \partial_{1}{ }^{2} \dot{\varphi} \ddot{\varphi}+a \dot{a} \partial_{2}{ }^{2} \dot{\varphi} \ddot{\varphi} \\
& \left.-a^{2} \dot{a}^{2} \ddot{\varphi} \ddot{\varphi}-\dot{a}^{2} \ddot{\varphi}\left(2 \partial_{1}{ }^{2} \varphi+2 \partial_{1}{ }^{2} \varphi-a \dot{a} \dot{\varphi}\right)\right),
\end{aligned}
$$

where $i$ should be summed from 1 to 3 . And $\left(\delta_{\theta} X\right)_{1}$ is

$$
\left(\delta_{\theta} X\right)_{1}=-\frac{1}{8}\left(\theta^{12}\right)^{2} H a \dot{a} \dot{\phi}_{0}\left(\partial_{1}^{2} \dot{\varphi}+\partial_{2}^{2} \dot{\varphi}-2 H \partial_{1}^{2} \varphi-2 H \partial_{2}^{2} \varphi+2 H a \dot{a} \dot{\varphi}\right) .
$$

Following the same procedure, we decompose $V$ as

$$
\delta_{\theta} V=\left(\delta_{\theta} V\right)_{3}+\left(\delta_{\theta} V\right)_{2}+\left(\delta_{\theta} V\right)_{1}+\left(\delta_{\theta} V\right)_{0}
$$

According to the same reason as that of the case of $\delta_{\theta} X$, we can just consider the leading terms in $\left(\delta_{\theta} V\right)_{2}$ and $\left(\delta_{\theta} V\right)_{1}$, which are

$$
\begin{aligned}
\left(\delta_{\theta} V\right)_{2}= & -\frac{n(n-1)}{8}\left(\theta^{12}\right)^{2} \phi_{0}^{n-2}\left(\partial_{1}^{2} \varphi \partial_{2}^{2} \varphi-\partial_{1} \partial_{2} \varphi \partial_{1} \partial_{2} \varphi+(a \dot{a})^{2} \dot{\varphi}^{2}-\right. \\
& \left.a \dot{a} \dot{\varphi} \partial_{1}^{2} \varphi-a \dot{a} \dot{\varphi} \partial_{2}^{2} \varphi\right), \\
\left(\delta_{\theta} V\right)_{1}=- & \frac{n(n-1)}{8}\left(\theta^{12}\right)^{2} \phi_{0}^{n-2}\left(2(a \dot{a})^{2} \dot{\phi}_{0} \dot{\varphi}-a \dot{a} \dot{\phi}_{0} \partial_{1}^{2} \varphi-a \dot{a} \dot{\phi}_{0} \partial_{2}^{2} \varphi\right) .
\end{aligned}
$$

The whole change of third order action of perturbation due to noncommutative geometry in leading order of slow-roll parameter can be written as

$$
\begin{aligned}
\delta_{\theta} S_{3}=\int d^{4} x \sqrt{h} & \left(P_{, X_{0} X_{0}}\left(\delta_{g} X\right)_{1}\left(\delta_{\theta} X\right)_{2}+P_{, X_{0} X_{0}}\left(\delta_{g} X\right)_{2}\left(\delta_{\theta} X\right)_{1}\right. \\
& +P_{, X_{0} \phi_{0}} \varphi\left(\delta_{\theta} X\right)_{2}+\frac{1}{2} P_{, X_{0} X_{0} X_{0}}\left(\delta_{g} X\right)_{1}^{2}\left(\delta_{\theta} X\right)_{1} \\
& \left.+P_{, V_{0} X_{0}}\left(\delta_{g} X\right)_{1}\left(\delta_{\theta} V\right)_{2}+P_{, V_{0} X_{0}}\left(\delta_{g} X\right)_{2}\left(\delta_{\theta} V\right)_{1}\right)
\end{aligned}
$$


where $\delta_{g} X=X-X_{0}$, and $\left(\delta_{g} X\right)_{1}=\dot{\phi}_{0} \dot{\varphi},\left(\delta_{g} X\right)_{2}=\frac{1}{2}\left(\dot{\varphi}^{2}-(\partial \varphi)^{2}\right)$, where we have picked out the terms with least $\dot{\phi}_{0}$ to reduce the order of slow-roll parameter. Although we have picked out the leading order terms, the result is still lengthy. We write the result of (3.14) in Appendix A. To write the result, we define two new parameters,

$$
\begin{gathered}
c_{V}^{2}=\frac{P_{, X_{0}}}{P_{, X_{0}}+2 V_{0} P_{, X_{0} V_{0}}}, \\
\sigma=X_{0} X_{0} X_{0} P_{, X_{0} X_{0} X_{0}} .
\end{gathered}
$$

As we pointed out above, all the terms except those with $\sigma$ coefficient are of order $O(\epsilon)$ in $\zeta$ after a gauge transformation $\varphi=\frac{\dot{\phi}}{H} \zeta$. These two parameters vanish in the general "slow-roll" inflation models, but could be non-trivial in some particular models. We will see in some case, they determine the dominance of the correction of non-Gaussianity.

There are some subtle differences between calculating the modification of threepoint function and two-point function due to noncommutativity. In calculating twopoint function, one has to solve the equation of motion of perturbation which is in general hard to solve with the presence of noncommutative coordinates(for a solvable example, see [38]). In Ref.[30], the author developed another way to encode the noncommutative effect into the power spectrum without solving the equation of motion.

However, even with a solvable equation, we do not need the modified classical solution to calculate noncommutative correction of three-point function. The threepoint function is calculated through

$$
\left\langle\zeta^{3}(t)\right\rangle=-i \int_{t_{0}}^{t} d t^{\prime}\left\langle\left[\zeta^{3}(t), H_{\text {int }}\left(t^{\prime}\right)\right]\right\rangle
$$

in tree level. So, to evaluate the modification of three-point function, which is denoted by $\left\langle\zeta^{3}(t)\right\rangle_{\theta}$ below, in the lowest order of $\theta$, we divide the Poisson bracket into two groups:

$$
\left\langle\zeta^{3}(t)\right\rangle_{\theta}=-i \int_{t_{0}}^{t} d t^{\prime}\left\langle\left[\zeta_{c}^{3}(t), \delta_{\theta} H_{\text {int }}\left(t^{\prime}\right)\right]\right\rangle-i \int_{t_{0}}^{t} d t^{\prime}\left\langle\left[3 \zeta_{c}^{2}(t) \delta_{\theta} \zeta(t), H_{\text {int }}\left(t^{\prime}\right)\right]\right\rangle,
$$

where $\zeta_{c}$ is the commutative solution. Since the primordial Hamiltonian without noncommutative modification is of order $\epsilon^{2}$ in slow-roll parameter, thus the second 
group contributes terms subleading in slow-roll parameter, as we emphasize the leading order of the modification part of Hamiltonian is $O(\epsilon)$. While the order of $\theta$ are $\theta^{2}$ in both case, so the leading modification is obtained from the first group of Poisson bracket which is summarized in Appendix B.

Another point deserved mention is that the constraint equations of $N$ and $N_{i}$, and thus the solutions (2.11) (2.12) obtain corrections of order $\theta^{2}$ in noncommutative coordinate. However, the same as the above analysis, they contribute terms of subleading order of $\theta$ or $\epsilon$ either in $\delta_{\theta} S$ or $S$ and thus we do not need to consider them.

\section{$4 \quad$ Model testing}

In this section, we evaluate the effect of noncommutativity on the non-Gaussianities of perturbation in some particular models. Note all the background field and Hubble scale are estimated at horizon crossing, namely at the time about 60 e-foldings before ending of inflation.

In the ordinary "slow-roll" inflation with Lagrangian $P=X-V$, since

$$
c_{s}=1, \quad c_{V}=1, \quad \sigma=0,
$$

it turns out that the modification is zero on the level of first order of slow-roll parameter. Let us turn to the models with significant non-Gaussianity.

\subsection{DBI model}

DBI inflation [11, 12, 13, 14] is motivated by brane inflation scenario[15, 16, 17, 18, 19] in warped compactifications. The effective Lagrangian is

$$
P(X, \phi)=-f(\phi)^{-1} \sqrt{1-2 f(\phi) X}+f(\phi)^{-1}-V(\phi),
$$

where $f$ is the warp factor $f=\frac{\lambda}{\phi^{4}}$, and $\lambda$ depends on flux number. The value of speed of sound $c_{s}$, as well as other two parameters $c_{f}$ ( $f$ substituting for $V$ in (3.15) represents warp factor $f(\phi))$ and $\sigma$ are as follows,

$$
\begin{aligned}
c_{s} & =\sqrt{1-\dot{\phi}^{2} f(\phi)}, \\
c_{f} & =\sqrt{1-\dot{\phi}^{2} f(\phi)}, \\
\sigma & =-\frac{3}{8} \dot{\phi}^{6} f^{2}\left(1-\dot{\phi}^{2} f\right)^{-\frac{5}{2}} .
\end{aligned}
$$


We find $c_{f}$ coincides with $c_{s}$ in DBI model, and thus it becomes as small as the speed of sound. The leading correction terms with respect to $c_{s}$ comes from (B.1) are

$$
\begin{aligned}
\left\langle\zeta_{k_{1}} \zeta_{k_{2}} \zeta_{k_{3}}\right\rangle_{\theta}= & i(2 \pi)^{3} \delta\left(\vec{k}_{1}+\vec{k}_{2}+\vec{k}_{3}\right) \frac{H^{4} l_{s}^{4}}{32 \epsilon^{2} c_{s}^{4} M_{p l}^{4}} \frac{1}{\prod\left(2 k_{i}^{3}\right)}\left[2 i \frac { H ^ { 4 } } { c _ { s } ^ { 4 } } \left(\left(k_{1}^{a}\right)^{2}\left(k_{2}^{b}\right)^{2}\right.\right. \\
& \left.-k_{1}^{a} k_{1}^{b} k_{2}^{a} k_{2}^{b}\right)\left(\vec{k}_{1} \cdot \vec{k}_{2}\right) k_{3}^{2}\left(24 \frac{1}{K^{5}}+120 \frac{k_{1}+k_{2}}{K^{6}}+720 \frac{k_{1} k_{2}}{K^{7}}\right) \\
& +4 \frac{i H^{3}}{c_{f}^{2} c_{s}^{2}} \frac{\phi_{0}}{\sqrt{\lambda}}\left(\left(k_{1}^{a}\right)^{2}\left(k_{2}^{b}\right)^{2}-k_{1}^{a} k_{1}^{b} k_{2}^{a} k_{2}^{b}\right)\left(\vec{k}_{1} \cdot \vec{k}_{2}\right)\left(8 \frac{1}{K^{3}}\right. \\
& \left.+24 \frac{k_{1} k_{2}+k_{2} k_{3}+k_{3} k_{1}}{K^{5}}+120 \frac{k_{1} k_{2} k_{3}}{K^{6}}\right) \\
& -6 i H^{2} \frac{1}{c_{f}^{2}} \frac{\phi_{0}^{2}}{\lambda}\left(( ( k _ { 1 } ^ { a } ) ^ { 2 } + ( k _ { 1 } ^ { b } ) ^ { 2 } ) k _ { 2 } k _ { 3 } \left(2 \frac{1}{K}\right.\right. \\
& \left.+2 \frac{k_{1} k_{2}+k_{2} k_{3}+k_{3} k_{1}}{K^{3}}+6 \frac{k_{1} k_{2} k_{3}}{K^{4}}\right)+2\left(\left(k_{1}^{a}\right)^{2}\left(k_{2}^{b}\right)^{2}\right. \\
& \left.\left.-k_{1}^{a} k_{1}^{b} k_{2}^{a} k_{2}^{b}\right) k_{3}^{2}\left(2 \frac{1}{K^{3}}+6 \frac{k_{1}+k_{2}}{K^{4}}+24 \frac{k_{1} k_{2}}{K^{5}}\right)\right) \\
& -i \frac{4 \sigma c_{s}^{2} H^{2}}{M_{p l}^{2} \epsilon}\left(\left(k_{1}^{a}\right)^{2}+\left(k_{1}^{b}\right)^{2}\right)\left(24 k_{1}^{2} k_{2}^{2} k_{3}^{2} \frac{1}{K^{5}}\right. \\
& \left.\left.-2 k_{2}^{2} k_{3}^{2}\left(2 \frac{1}{K^{3}}+6 \frac{k_{1}}{K^{4}}\right)\right)+p e r m .\right]
\end{aligned}
$$

where $a$ and $b$ denote the first and second component of $k$ vector respectively, $K=$ $k_{1}+k_{2}+k_{3}$ and perm. denotes all the other terms obtained by rotating the index $(1,2,3)$. Here we have used the relation $\dot{\phi}_{0}^{2} \simeq \frac{\phi_{0}^{4}}{\lambda}$ due to small speed of sound. In this phase, we have power spectrum of $\phi$ perturbation

$$
P_{k}=\frac{H^{4}}{4 \pi^{2} \dot{\phi}_{0}^{2}} \approx \frac{N_{e}^{4}}{4 \pi^{2} \lambda},
$$

where $N_{e} \sim 60$ is the e-folding between horizon crossing and the end of inflation. According to COBE normalization, $P_{k} \approx 23 \times 10^{-10}$, we find $\lambda \approx 10^{14}$ and $\dot{\phi}_{0} \approx$ $10^{-7} M_{p l}^{2}$. Using the limit of small speed of sound, $\phi_{0} \approx \dot{\phi}_{0}^{1 / 2} \lambda^{1 / 4} \approx M_{p l}$. As a matter of result, only the first term in (4.6) with the following shape dominates,

$$
\begin{aligned}
\mathcal{A}_{1}= & -\frac{H^{4} l_{s}^{4}}{32 c_{s}^{6}}\left(\left(k_{1}^{a}\right)^{2}\left(k_{2}^{b}\right)^{2}-k_{1}^{a} k_{1}^{b} k_{2}^{a} k_{2}^{b}\right)\left(\vec{k}_{1} \cdot \vec{k}_{2}\right) k_{3}^{2}\left(24 \frac{1}{K^{5}}\right. \\
& \left.+120 \frac{k_{1}+k_{2}}{K^{6}}+720 \frac{k_{1} k_{2}}{K^{7}}\right)+ \text { perm. }
\end{aligned}
$$

We parameterize $\left\langle\zeta_{k_{1}} \zeta_{k_{2}} \zeta_{k_{3}}\right\rangle_{\theta}$ as,

$$
\left\langle\zeta_{k_{1}} \zeta_{k_{2}} \zeta_{k_{3}}\right\rangle_{\theta}=(2 \pi)^{7} \delta\left(\vec{k}_{1}+\vec{k}_{2}+\vec{k}_{3}\right)\left(P_{\zeta}^{k}\right)^{2} \frac{1}{\prod_{i} k_{i}^{3}} \mathcal{A}_{1}
$$




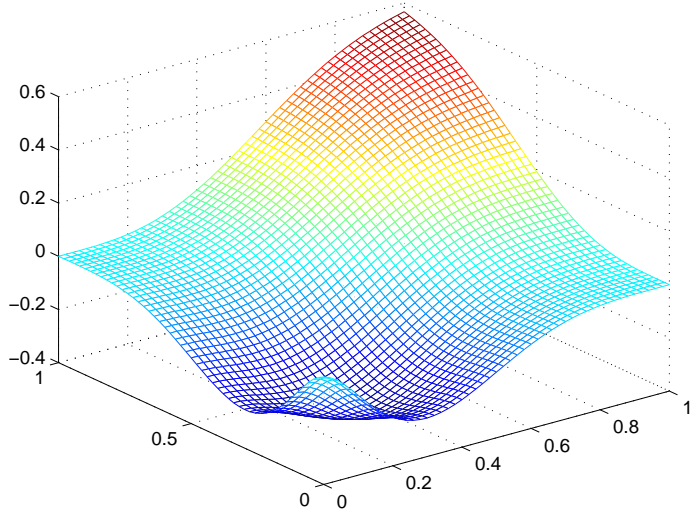

Figure 1: The shape of $\mathcal{A}_{1} / k_{1} k_{2} k_{3}$.

where $P_{\zeta}^{k}$ is the primordial power spectrum [20]

$$
P_{\zeta}^{k}=\frac{1}{8 \pi^{2} M_{p l}^{2}} \frac{H^{2}}{c_{s} \epsilon}
$$

The shape of $\mathcal{A}_{1} / k_{1} k_{2} k_{3}$ as function of $x_{1}=k_{1} / k_{3}$ and $x_{2}=k_{2} / k_{3}$ are drawn in Fig. 1. In drawing the figures, we omit the coefficient of $\mathcal{A}_{1}$. And we have chosen a particular frame in which the three $\vec{k}$ modes are in $x-y$ plane and $\vec{k}_{3}$ is along the $x$-axis. We find this shape is distinct from the shape of $\mathcal{A}_{c}[7]$.

The non-Gaussianity of CMB in the WMAP observations is analyzed by assuming the following ansatz

$$
\zeta=\zeta_{g}-\frac{3}{5} f_{N L} \zeta_{g}^{2}
$$

where $\zeta_{g}$ represents the Gaussian part of $\zeta$, and $f_{N L}$ is an estimator of non-Gaussianity. The three-point function of $\zeta$ can be factorized as

$$
\left\langle\zeta_{\vec{k}_{1}} \zeta_{\vec{k}_{2}} \zeta_{\vec{k}_{3}}\right\rangle=(2 \pi)^{7} \delta^{3}\left(\vec{k}_{1}+\vec{k}_{2}+\vec{k}_{3}\right)\left(-\frac{3}{10} f_{N L}\left(P_{\zeta}^{k}\right)^{2}\right) \frac{\sum_{i} k_{i}^{3}}{\prod_{i} k_{i}^{3}} .
$$

Despite the difference between the shape of (4.6) and that of (4.12), we set $k_{1}=$ $k_{2}=k_{3}=k$ to calculate $f_{N L}^{1}$ which represents the size of correction of non-Gaussianity deriving from $\mathcal{A}_{1}$. We have

$$
f_{N L}^{1}=0.02 \frac{H^{4} l_{s}^{4}}{c_{s}^{6}}
$$

where the results are evaluated in the particular frame we used above. 
We find the noncommutative correction of non-Gaussianity could be large if $c_{s} \ll$ 1. For example, for Hubble constant $H \sim 10^{-5} M_{p l}$, the string length scale $l_{s} \sim$ $10^{4} M_{p l}^{-1}$ and the speed of sound $c_{s} \sim 0.1$, then $f_{N L}^{1} \sim 2$. Comparing to the dominant $f_{N L}$ of commutative case $((5.10)$ in [7]),

$$
f_{N L}^{c} \approx 0.32 c_{s}^{-2}
$$

we find the correction is more sensitive to the speed of sound $c_{s}$. The present observation imposes bound on equilateral form of $f_{N L},-256<f_{N L}<332$, and future observation of Planck can detect $\left|f_{N L}\right| \gtrsim 5$, thus making the correction within the sensibility of these observation.

\subsection{K-inflation}

We now consider the correction in K-inflation[20, 21] model which also has small speed of sound. The Lagrangian of the power law K-inflation is of the form

$$
P(X, \phi)=\frac{4}{9} \frac{4-3 \gamma}{\gamma^{2}} \frac{1}{\phi^{2}}\left(-X+X^{2}\right),
$$

where $\gamma$ is a constant. In the inflationary solution, $X$ remains constant as

$$
X_{0}=\frac{2-\gamma}{4-3 \gamma}
$$

The solution leads to scale factor $a$ of

$$
a \sim t^{\frac{2}{3 \gamma}}
$$

for any $0<\gamma<\frac{2}{3}$. And the speed of sound is

$$
c_{s}^{2}=\frac{\gamma}{8-3 \gamma}
$$

In order to get small speed of sound, we focus on the region $\gamma \ll 1$. The power spectrum in the limit of small $\gamma$ is [20]

$$
P_{\zeta}^{k}=\frac{1}{c_{s}} \frac{2}{3 \gamma} \frac{H^{2}}{8 \pi^{2} M_{p l}^{2}}\left(\frac{k}{k_{1}}\right)^{-3 \gamma},
$$

where $H$ is taken to be the Hubble scale at the time of horizon exit for the perturbations currently at our horizon, and $k_{1}$ is the associated comoving wavenumber. Using (4.18) as well as the fact that data determines $P_{\zeta}^{k} \sim 10^{-9}$ at horizon crossing, we get

$$
H^{2} \sim \frac{3}{4 \sqrt{2}} \gamma^{3 / 2} 8 \pi^{2} M_{p l}^{2} \times 10^{-9}
$$


It follows from (4.20) that the tilt satisfies

$$
n_{s}-1=-3 \gamma+\cdots
$$

which allows us to determine $\gamma \sim \frac{1}{60}$ and thus $c_{s}^{2} \sim \frac{1}{480}$ using the central value of the spectral index in the WMAP results[2].

We also find $c_{V}=-1$ and $\sigma=0$ in this model, where we choose $V=\phi^{2}$. The dominant terms in three-point function are those with most $c_{s}$ in the denominator in each kind of coefficients,

$$
\begin{aligned}
\left\langle\zeta_{k_{1}} \zeta_{k_{2}} \zeta_{k_{3}}\right\rangle_{\theta}= & i(2 \pi)^{3} \delta\left(\vec{k}_{1}+\vec{k}_{2}+\vec{k}_{3}\right) \frac{H^{4} l_{s}^{4}}{32 \epsilon^{2} c_{s}^{4} M_{p l}^{4}} \frac{1}{\prod\left(2 k_{i}^{3}\right)}\left[2 i \frac { H ^ { 4 } } { c _ { s } ^ { 4 } } \left(\left(k_{1}^{a}\right)^{2}\left(k_{2}^{b}\right)^{2}\right.\right. \\
& \left.-k_{1}^{a} k_{1}^{b} k_{2}^{a} k_{2}^{b}\right)\left(\vec{k}_{1} \cdot \vec{k}_{2}\right) k_{3}^{2}\left(24 \frac{1}{K^{5}}+120 \frac{k_{1}+k_{2}}{K^{6}}+720 \frac{k_{1} k_{2}}{K^{7}}\right) \\
& -2 \frac{i H^{3}}{c_{s}^{2}} \frac{M_{p l}^{2}}{\phi_{0}}\left(\left(k_{1}^{a}\right)^{2}\left(k_{2}^{b}\right)^{2}-k_{1}^{a} k_{1}^{b} k_{2}^{a} k_{2}^{b}\right)\left(\vec{k}_{1} \cdot \vec{k}_{2}\right)\left(8 \frac{1}{K^{3}}\right. \\
& \left.+24 \frac{k_{1} k_{2}+k_{2} k_{3}+k_{3} k_{1}}{K^{5}}+120 \frac{k_{1} k_{2} k_{3}}{K^{6}}\right) \\
& +i H^{2} \frac{M_{p l}^{4}}{\phi_{0}^{2}}\left(( ( k _ { 1 } ^ { a } ) ^ { 2 } + ( k _ { 1 } ^ { b } ) ^ { 2 } ) ( \vec { k } _ { 2 } \cdot \vec { k } _ { 3 } ) \left(2 \frac{1}{K}+2 \frac{k_{1} k_{2}+k_{2} k_{3}+k_{3} k_{1}}{K^{3}}\right.\right. \\
& \left.+6 \frac{k_{1} k_{2} k_{3}}{K^{4}}\right)+2\left(\left(k_{1}^{a}\right)^{2}\left(k_{2}^{b}\right)^{2}-k_{1}^{a} k_{1}^{b} k_{2}^{a} k_{2}^{b}\right) k_{3}^{2}\left(2 \frac{1}{K^{3}}\right. \\
& \left.\left.+6 \frac{k_{1}+k_{2}}{K^{4}}+24 \frac{k_{1} k_{2}}{K^{5}}\right)\right)+ \text { perm. } .
\end{aligned}
$$

Following the same parametrization as (4.9), we get

$$
\begin{gathered}
\mathcal{A}_{1}=-\frac{H^{4} l_{s}^{4}}{32 c_{s}^{6}}\left(\left(k_{1}^{a}\right)^{2}\left(k_{2}^{b}\right)^{2}-k_{1}^{a} k_{1}^{b} k_{2}^{a} k_{2}^{b}\right)\left(\vec{k}_{1} \cdot \vec{k}_{2}\right) k_{3}^{2}\left(24 \frac{1}{K^{5}}\right. \\
\left.+120 \frac{k_{1}+k_{2}}{K^{6}}+720 \frac{k_{1} k_{2}}{K^{7}}\right)+ \text { perm. } \\
\mathcal{A}_{2}=\frac{H^{3} M_{p l}^{2} l_{s}^{4}}{32 c_{s}^{4} \phi_{0}}\left(\left(k_{1}^{a}\right)^{2}\left(k_{2}^{b}\right)^{2}-k_{1}^{a} k_{1}^{b} k_{2}^{a} k_{2}^{b}\right)\left(\vec{k}_{1} \cdot \vec{k}_{2}\right)\left(8 \frac{1}{K^{3}}\right. \\
\left.\quad+24 \frac{k_{1} k_{2}+k_{2} k_{3}+k_{3} k_{1}}{K^{5}}+120 \frac{k_{1} k_{2} k_{3}}{K^{6}}\right)+ \text { perm. }, \\
\mathcal{A}_{3}=-\frac{H^{2} M_{p l}^{4} l_{s}^{4}}{64 c_{s}^{2} \phi_{0}^{2}}\left(( ( k _ { 1 } ^ { a } ) ^ { 2 } + ( k _ { 1 } ^ { b } ) ^ { 2 } ) ( \vec { k } _ { 2 } \cdot \vec { k } _ { 3 } ) \left(2 \frac{1}{K}+2 \frac{k_{1} k_{2}+k_{2} k_{3}+k_{3} k_{1}}{K^{3}}\right.\right. \\
\left.+6 \frac{k_{1} k_{2} k_{3}}{K^{4}}\right)+2\left(\left(k_{1}^{a}\right)^{2}\left(k_{2}^{b}\right)^{2}-k_{1}^{a} k_{1}^{b} k_{2}^{a} k_{2}^{b}\right) k_{3}^{2}\left(2 \frac{1}{K^{3}}+6 \frac{k_{1}+k_{2}}{K^{4}}\right. \\
\left.\left.+24 \frac{k_{1} k_{2}}{K^{5}}\right)\right)+ \text { perm.. }
\end{gathered}
$$


The shape of $\mathcal{A}_{1} / k_{1} k_{2} k_{3}$ is the same as that of DBI case. The shape of $\mathcal{A}_{2} / k_{1} k_{2} k_{3}$ and $\mathcal{A}_{3} / k_{1} k_{2} k_{3}$ are drawn in Fig. 2 and Fig. 3 respectively.

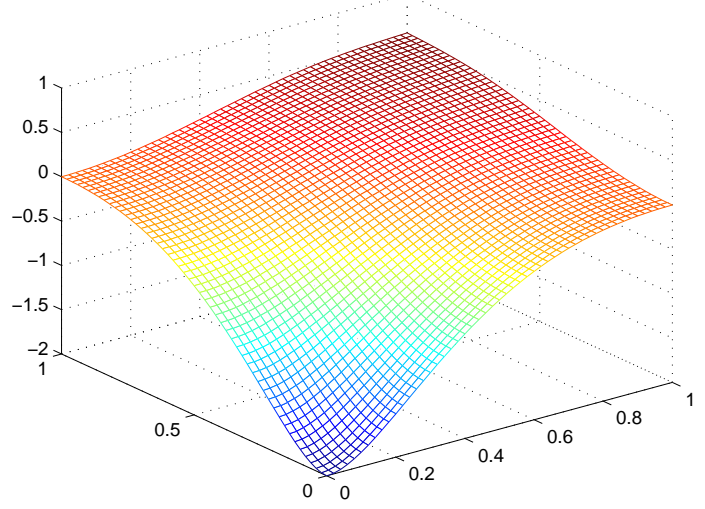

Figure 2: The shape of $\mathcal{A}_{2} / k_{1} k_{2} k_{3}$.

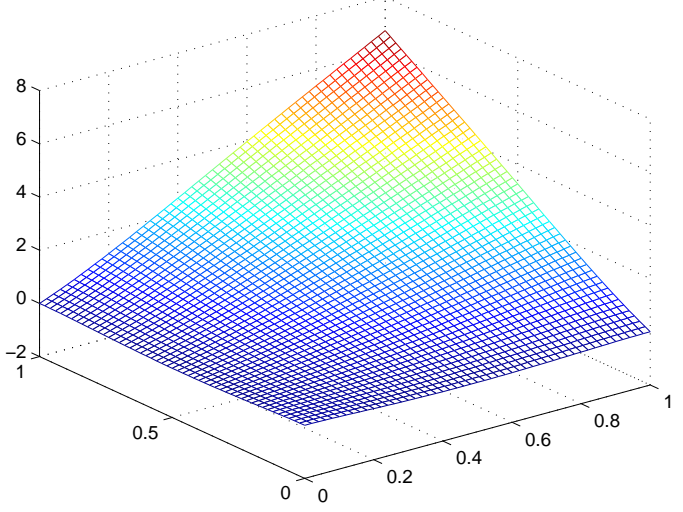

Figure 3: The shape of $\mathcal{A}_{3} / k_{1} k_{2} k_{3}$.

In the equilateral triangle configuration $k_{1}=k_{2}=k_{3}=k$, the correction of $f_{N L}$ is divided into $f_{N L}^{1}, f_{N L}^{2}, f_{N L}^{3}$,

$$
\begin{array}{r}
f_{N L}^{1}=0.02 \frac{H^{4} l_{s}^{4}}{c_{s}^{6}}, \\
f_{N L}^{2}=-0.02 \frac{H^{3} l_{s}^{4} M_{p l}^{2}}{\phi_{0} c_{s}^{4}}, \\
f_{N L}^{3}=0.25 \frac{H^{2} l_{s}^{4} M_{p l}^{4}}{\phi_{0}^{2} c_{s}^{2}},
\end{array}
$$


We estimate the size of $\phi_{0}$ through Friedmann equation

$$
3 M_{p l}^{2} H^{2}=E,
$$

where

$$
E=2 X P_{, X}-P=\frac{4}{9} \frac{4-3 \gamma}{\gamma^{2}} \frac{1}{\phi^{2}}\left(-X+3 X^{2}\right),
$$

and find $\phi_{0} \approx \frac{M_{p l}^{2}}{12 \sqrt{3} c_{s}^{2} H}$. Using (4.18) and (4.20), we find

$$
f_{N L}^{2}=-12 \sqrt{3} c_{s}^{4} f_{N L}^{1}
$$

and

$$
f_{N L}^{3}=5400 c_{s}^{8} f_{N L}^{1}
$$

Since $c_{s}^{2} \sim \frac{1}{480}$, these two are much smaller than $f_{N L}^{1}$. Using (4.18), (4.20) and $l_{s}=10^{4} M_{p l}^{-1}$, we find

$$
f_{N L}^{1} \approx 180
$$

which does not depend on $c_{s}$ due to the cancelation between $H^{4}$ and $c_{s}^{6}$. This property is different from the case of DBI model. The dominant non-Gaussianity in K-inflation without considering noncommutative effect is

$$
f_{N L}^{c} \approx 0.26 c_{s}^{-2} \approx 125 .
$$

Since $c_{s}$ is fixed in K-inflation, it is easy to set an upper bound on $l_{s}$. Adding (4.26) and (4.34) together, we get the total non-gaussianity in K-inflation,

$$
f_{N L}^{t}=180 \times 10^{-16} l_{s}^{4} M_{p l}^{4}+125 .
$$

Using the upper bound on the equilateral form of $f_{N L}, f_{N L}<332$, we get

$$
l_{s} \leqslant 10^{4} M_{p l}^{-1} .
$$

\section{Conclusion}

We studied the noncommutative corrections of non-Gaussianities of primordial perturbation in a general framework. The corrections could be large in the models with a small speed of sound and a relatively low string scale. We test our result in two 
particular models, DBI model and K-inflation. We find that the correction of $f_{N L}$ can reach $O(1)$ or even bigger, and thus is observable within the sensibility of future experiments. Our study also shows that the noncommutative corrections are more sensitive to the speed of sound than the usual nonGaussianity estimator. This could be a clue to distinguish the different contributions to the non-Gaussianity. And also it indicates that in the inflation models with large speed of sound, the noncommutative correction to the nonGaussianity is small. Moreover, the shape of the corrections are different from the commutative case, which can be used as another distinguishing estimator.

From our study, it turns out that the noncommutative corrections become significant when the string scale is relatively low. This could be just an illusion, given the noncommutative scale may not be string scale. In fact, the leading order correction terms are actually proportional to $\left(\theta^{12}\right)^{2}$, which could be nontrivially related to string scale. If we have a relatively low noncommutative scale, namely we assume a little larger noncommutativity, the correction could be larger. In string theory, the noncommutative scale depends also on the background $B_{12}$ field on D-brane. In a sense, the corrections tell us the information of the string scale and also of the background field.

On the other hand, without assuming another scale, one can make the natural choice (1.5). Although it is not easy to explain the great difference between the Planck scale and the string scale in the perturbative string theory, it is not hard to tune the string length scale all the way up to $10^{-18} \mathrm{~cm}$ in type I compactifications and nonperturbative heterotic string theory. More interestingly, in recent phenomenology study, the string scale is fit by WMAP data to be around $10^{-5} \sim 10^{-4} M_{p l}$ [31, 32, 33. Doubtlessly, future experiment will bring forward more data to test this tentative choice.

\section{Acknowledgments}

The work was partially supported by NSFC Grant No. 10405028,10535060, NKBRPC (No. 2006CB805905) and the Key Grant Project of Chinese Ministry of Education (NO. 305001). K. Fang thanks S.-H. Henry Tye for helpful suggestion and Gary Shiu 
for discussion.

\section{A Noncommutative correction of third order per- turbation}

The noncommutative correction of third order action of perturbation, which is of order $O(\epsilon)$ except the terms proportional to $\sigma$, is

$$
\begin{aligned}
\delta_{\theta} S=\int & d^{4} x a^{3} \frac{1}{8}\left(\theta^{12}\right)^{2}\left(( \frac { P _ { , X _ { 0 } } } { \dot { \phi } _ { 0 } } ( \frac { 1 } { c _ { s } ^ { 2 } } - 1 ) \dot { \varphi } + P _ { , X _ { 0 } } \frac { V _ { 0 } ^ { \prime } } { 2 V _ { 0 } } ( \frac { 1 } { c _ { V } ^ { 2 } } - 1 ) \varphi ) \left(\frac{1}{a^{2}} \partial_{1}^{2} \partial_{i} \varphi \partial_{2}^{2} \partial_{i} \varphi\right.\right. \\
& -\frac{1}{a^{2}} \partial_{1} \partial_{2} \partial_{i} \varphi \partial_{1} \partial_{2} \partial_{i} \varphi-H \partial_{1}^{2} \partial_{i} \varphi \partial_{i} \dot{\varphi}-H \partial_{2}^{2} \partial_{i} \varphi \partial_{i} \dot{\varphi}+H^{2} \partial_{1}^{2} \partial_{i} \varphi \partial_{i} \varphi+ \\
& H^{2} \partial_{2}^{2} \partial_{i} \varphi \partial_{i} \varphi+\dot{a}^{2} \partial_{1} \dot{\varphi} \partial_{1} \dot{\varphi}+\dot{a}^{2} \partial_{2} \dot{\varphi} \partial_{2} \dot{\varphi}+\dot{a}^{2} \partial_{i} \dot{\varphi} \partial_{i} \dot{\varphi}-2 H \dot{a}^{2} \partial_{1} \dot{\varphi} \partial_{1} \varphi \\
& -2 H \dot{a}^{2} \partial_{2} \dot{\varphi} \partial_{2} \varphi-2 H \dot{a}^{2} \partial_{i} \dot{\varphi} \partial_{i} \varphi+(H \dot{a})^{2} \partial_{1} \varphi \partial_{1} \varphi+(H \dot{a})^{2} \partial_{2} \varphi \partial_{2} \varphi \\
& +(H \dot{a})^{2} \partial_{i} \varphi \partial_{i} \varphi-\partial_{1}^{2} \dot{\varphi} \partial_{2}^{2} \dot{\varphi}+\partial_{1} \partial_{2} \dot{\varphi} \partial_{1} \partial_{2} \dot{\varphi}-(H a \dot{a})^{2} \dot{\varphi}^{2}-H a \dot{a} \dot{\varphi} \partial_{1}^{2} \dot{\varphi} \\
& -H a \dot{a} \dot{\varphi} \partial_{2}^{2} \dot{\varphi}+2 H \partial_{1}^{2} \dot{\varphi} \partial_{2}^{2} \varphi+2 H \partial_{1}^{2} \varphi \partial_{2}^{2} \dot{\varphi}+2 H^{2} a \dot{a} \dot{\varphi} \partial_{1}^{2} \varphi+2 H^{2} a \dot{a} \dot{\varphi} \partial_{2}^{2} \varphi \\
& -4 H \partial_{1} \partial_{2} \dot{\varphi} \partial_{1} \partial_{2} \varphi-4 H^{2} \partial_{1}^{2} \varphi \partial_{2}^{2} \varphi+4 H^{2} \partial_{1} \partial_{2} \varphi \partial_{1} \partial_{2} \varphi+a \dot{a} \partial_{1}^{2} \dot{\varphi} \ddot{\varphi} \\
& \left.+a \dot{a} \partial_{2}^{2} \dot{\varphi} \ddot{\varphi}-a^{2} \dot{a}^{2} \ddot{\varphi} \ddot{\varphi}-\dot{a}^{2} \ddot{\varphi}\left(2 \partial_{1}{ }^{2} \varphi+2 \partial_{1}{ }^{2} \varphi-a \dot{a} \dot{\varphi}\right)\right)-\frac{1}{2} \frac{P, X_{0}}{\dot{\phi}_{0}}\left(\frac{1}{c_{s}^{2}}-1\right) H a \dot{a} \\
& \left(\dot{\varphi}^{2}-(\partial \varphi)^{2}\right)\left(\partial_{1}^{2} \dot{\varphi}+\partial_{2}^{2} \dot{\varphi}-2 H \partial_{1}^{2} \varphi-2 H \partial_{2}^{2} \varphi+2 H a \dot{a} \dot{\varphi}\right) \\
& \left.-P_{, X_{0}} \frac{n(n-1) \dot{\phi}_{0}}{4 \phi_{0}^{2}}\left(\frac{1}{c_{V}^{2}}-1\right)\left(\dot{\varphi}^{2}-(\partial \varphi)^{2}\right)\left(2(a \dot{a})^{2} \dot{\varphi}-a \dot{a} \partial_{1}^{2} \varphi-a \dot{a} \partial_{2}^{2} \varphi\right)\right) \\
& \left.-P_{, X_{0}} \frac{n(n-1) \dot{\phi}_{0}}{2 \phi_{0}^{2}}\left(\frac{1}{c_{V}^{2}}-1\right)\right)\left(\dot{\varphi} \partial_{1}^{2} \varphi \partial_{2}^{2} \varphi-\dot{\varphi} \partial_{1} \partial_{2} \varphi \partial_{1} \partial_{2} \varphi+(a \dot{a})^{2} \dot{\varphi} \dot{\varphi}^{3}\right. \\
& \left.-a \dot{a} \dot{\varphi}^{2} \partial_{1}^{2} \varphi-a \dot{a} \dot{\varphi}^{2} \partial_{2}^{2} \varphi\right)-\frac{\sigma}{X_{0}^{2}} H a \dot{a} \dot{\phi}_{0} \dot{\varphi}^{2}\left(\partial_{1}^{2} \dot{\varphi}+\partial_{2}^{2} \dot{\varphi}-2 H \partial_{1}^{2} \varphi-2 H \partial_{2}^{2} \varphi\right. \\
& +2 H a \dot{a} \dot{\varphi})) .
\end{aligned}
$$




\section{B Noncommutative correction of three-point func- tion}

The correction of three-point function of $\zeta$ is

$$
\begin{aligned}
& \left\langle\zeta_{\vec{k}_{1}} \zeta_{\vec{k}_{2}} \zeta_{\vec{k}_{3}}\right\rangle_{\theta}=i(2 \pi)^{3} \delta^{3}\left(\vec{k}_{1}+\vec{k}_{2}+\vec{k}_{3}\right) \frac{H^{4} l_{s}^{4}}{32 \epsilon^{2} c_{s}^{4} M_{p l}^{4}} \frac{1}{\prod\left(2 k_{i}^{3}\right)}\left(2 c_{s} H^{4}\left(\frac{1}{c_{s}^{2}}-1\right)\right. \\
& {\left[( ( k _ { 1 } ^ { a } ) ^ { 2 } ( k _ { 2 } ^ { b } ) ^ { 2 } - k _ { 1 } ^ { a } k _ { 1 } ^ { b } k _ { 2 } ^ { a } k _ { 2 } ^ { b } ) \left(\left(\vec{k}_{1} \cdot \vec{k}_{2}\right) k_{3}^{2} \frac{i}{c_{s}^{3}}\left(24 \frac{1}{K^{5}}+120 \frac{k_{1}+k_{2}}{K^{6}}+720 \frac{k_{1} k_{2}}{K^{7}}\right)\right.\right.} \\
& \left.-720 k_{1}^{2} k_{2}^{2} k_{3}^{2} \frac{i}{c_{s}} \frac{1}{K^{7}}-k_{3}^{2} \frac{i}{c_{s}}\left(8 \frac{1}{K^{3}}+24 \frac{k_{1}+k_{2}}{K^{4}}+96 \frac{k_{1} k_{2}}{K^{5}}\right)\right) \\
& +\left(\left(k_{1}^{a}\right)^{2}+\left(k_{1}^{b}\right)^{2}\right)\left(-\left(\vec{k}_{1} \cdot \vec{k}_{2}\right) k_{2}^{2} k_{3}^{2} \frac{i}{c_{s}}\left(24 \frac{1}{K^{5}}+120 \frac{k_{1}}{K^{6}}\right)\right. \\
& +\left(\vec{k}_{1} \cdot \vec{k}_{2}\right) k_{3}^{2} \frac{i}{c_{s}}\left(2 \frac{1}{K^{3}}+6 \frac{k_{1}+k_{2}}{K^{4}}+24 \frac{k_{1} k_{2}}{K^{5}}\right)-24 k_{1}^{2} k_{2}^{2} k_{3}^{2} c_{s}^{3} \frac{i}{K^{5}} \\
& +k_{2}^{2} k_{3}^{2} c_{s} i\left(4 \frac{1}{K^{3}}+12 \frac{k_{1}}{K^{4}}\right)+12 i k_{1}^{2} k_{2}^{2} k_{3}^{2} c_{s} \frac{1}{K^{5}} \\
& +i k_{2}^{2} k_{3}^{2} c_{s}\left(2 \frac{1}{K^{3}}+6 \frac{k_{1}}{K^{4}}\right)+k_{1}^{2}\left(\vec{k}_{2} \cdot \vec{k}_{3}\right) \frac{i}{c_{s}}\left(\frac{1}{K^{3}}+3 \frac{k_{2}+k_{3}}{K^{4}}+12 \frac{k_{2} k_{3}}{K^{5}}\right) \\
& \left.-\left(\vec{k}_{2} \cdot \vec{k}_{3}\right) \frac{i}{c_{s}}\left(\frac{2}{K}+2 \frac{k_{1} k_{2}+k_{2} k_{3}+k_{3} k_{1}}{K^{3}}+6 \frac{k_{1} k_{2} k_{3}}{K^{4}}\right)\right) \\
& +\left(k_{1}^{a} k_{2}^{a}+k_{1}^{b} k_{2}^{b}+\vec{k}_{1} \cdot \vec{k}_{2}\right)\left(24 i k_{1}^{2} k_{2}^{2} k_{3}^{2} c_{s} \frac{1}{K^{5}}-2 i k_{2}^{2} k_{3}^{2} c_{s}\left(2 \frac{1}{K^{3}}+6 \frac{k_{1}}{K^{4}}\right)\right. \\
& \left.+k_{3}^{2} i c_{s}\left(\frac{1}{K}+\frac{k_{1}+k_{2}}{K^{2}}+2 \frac{k_{1} k_{2}}{K^{3}}\right)\right)-4 i c_{s}^{3} k_{1}^{2} k_{2}^{2} k_{3}^{2} \frac{1}{K^{3}} \\
& +2\left(\left(k_{1}^{a}\right)^{2}\left(k_{2}^{b}\right)^{2}+\left(k_{1}^{b}\right)^{2}\left(k_{2}^{a}\right)^{2}\right) k_{2}^{2} k_{3}^{2} \frac{i}{c_{s}}\left(24 \frac{1}{K^{5}}+120 \frac{k_{1}}{K^{6}}\right) \\
& -4 k_{1}^{a} k_{1}^{b} k_{2}^{a} k_{2}^{b} k_{2}^{2} k_{3}^{2} \frac{i}{c_{s}}\left(24 \frac{1}{K^{5}}+120 \frac{k_{1}}{K^{6}}\right) \\
& \left.+\left(\vec{k}_{2} \cdot \vec{k}_{3}\right) k_{1}^{2} i c_{s}\left(\frac{1}{K}+\frac{k_{2}+k_{3}}{K^{2}}+2 \frac{k_{2} k_{3}}{K^{3}}\right)\right] \\
& -i k_{1}^{2} k_{2}^{2} k_{3}^{2} c_{s}\left[\left(k_{1}^{a}\right)^{2}+\left(k_{1}^{b}\right)^{2}\right]\left(\frac{48}{K^{5}}+\frac{120 k_{2}}{K^{6}}\right) \\
& +i k_{1}^{2} k_{2}^{2} k_{3}^{2} c_{s}^{3}\left(\frac{8}{K^{3}}-12 \frac{k_{1}+k_{2}}{K^{4}}+24 \frac{k_{1} k_{2}}{K^{5}}\right) \\
& +2 i k_{2}^{2} k_{3}^{2} c_{s}\left[\left(k_{1}^{a}\right)^{2}+\left(k_{1}^{b}\right)^{2}\right]\left(\frac{4}{K^{3}}-6 \frac{k_{2}-2 k_{1}}{K^{4}}-24 \frac{k_{1} k_{2}}{K^{5}}\right) \\
& -2 i k_{1}^{2} k_{2}^{2} k_{3}^{2} c_{s}^{3}\left(\frac{4}{K^{3}}-6 \frac{k_{2}}{K^{4}}\right) \\
& +n c_{s} H^{3}\left(\frac{1}{c_{V}^{2}}-1\right) \frac{\dot{\phi}_{0}}{\phi_{0}}\left[( ( k _ { 1 } ^ { a } ) ^ { 2 } ( k _ { 2 } ^ { b } ) ^ { 2 } - k _ { 1 } ^ { a } k _ { 1 } ^ { b } k _ { 2 } ^ { a } k _ { 2 } ^ { b } ) \left(( \vec { k } _ { 1 } \cdot \vec { k } _ { 2 } ) \frac { i } { c _ { s } ^ { 3 } } \left(8 \frac{1}{K^{3}}\right.\right.\right. \\
& \left.+24 \frac{k_{1} k_{2}+k_{2} k_{3}+k_{3} k_{1}}{K^{5}}+120 \frac{k_{1} k_{2} k_{3}}{K^{6}}\right)-k_{1}^{2} k_{2}^{2} \frac{i}{c_{s}}\left(24 \frac{1}{K^{5}}+120 \frac{k_{3}}{K^{6}}\right)
\end{aligned}
$$




$$
\begin{aligned}
& \left.-4 \frac{i}{c_{s}}\left(2 \frac{1}{K}+2 \frac{k_{1} k_{2}+k_{2} k_{3}+k_{3} k_{1}}{K^{3}}+6 \frac{k_{1} k_{2} k_{3}}{K^{4}}\right)\right) \\
& +\left(\left(k_{1}^{a}\right)^{2}+\left(k_{1}^{b}\right)^{2}\right)\left(-\left(\vec{k}_{1} \cdot \vec{k}_{2}\right) k_{2}^{2} \frac{i}{c_{s}}\left(2 \frac{1}{K^{3}}+6 \frac{k_{1}+k_{3}}{K^{4}}+24 \frac{k_{1} k_{3}}{K^{5}}\right)\right. \\
& +\left(\vec{k}_{1} \cdot \vec{k}_{2}\right) \frac{i}{c_{s}}\left(2 \frac{1}{K}+2 \frac{k_{1} k_{2}+k_{2} k_{3}+k_{3} k_{1}}{K^{3}}+6 \frac{k_{1} k_{2} k_{3}}{K^{4}}\right) \\
& \left.-k_{1}^{2} k_{2}^{2} i c_{s}\left(2 \frac{1}{K^{3}}+6 \frac{k_{3}}{K^{4}}\right)+2 k_{2}^{2} i c_{s}\left(\frac{1}{K}+\frac{k_{1}+k_{3}}{K^{2}}+2 \frac{k_{1} k_{3}}{K^{3}}\right)\right) \\
& -\left(k_{1}^{a} k_{2}^{a}+k_{1}^{b} k_{2}^{b}+\vec{k}_{1} \cdot \vec{k}_{2}\right)\left(-k_{1}^{2} k_{2}^{2} i c_{s}\left(2 \frac{1}{K^{3}}+6 \frac{k_{3}}{K^{4}}\right)\right. \\
& +2 k_{1}^{2} i c_{s}\left(\frac{1}{K}+\frac{k_{2}+k_{3}}{K^{2}}+2 \frac{k_{2} k_{3}}{K^{2}}\right) \\
& \left.-i c_{s}\left(K+\frac{k_{1} k_{2}+k_{2} k_{3}+k_{3} k_{1}}{K}+\frac{k_{1} k_{2} k_{3}}{K^{2}}\right)\right)-k_{2}^{2} k_{3}^{2} i c_{s}^{3}\left(\frac{1}{K}+\frac{k_{1}}{K^{2}}\right) \\
& +2\left(\left(k_{1}^{a}\right)^{2}\left(k_{2}^{b}\right)^{2}+\left(k_{1}^{b}\right)^{2}\left(k_{2}^{a}\right)^{2}\right) k_{1}^{2} \frac{i}{c_{s}}\left(2 \frac{1}{K^{3}}+6 \frac{k_{2}+k_{3}}{K^{4}}+24 \frac{k_{2} k_{3}}{K^{5}}\right) \\
& -4 k_{1}^{a} k_{1}^{b} k_{2}^{a} k_{2}^{b} k_{1}^{2} \frac{i}{c_{s}}\left(2 \frac{1}{K^{3}}+6 \frac{k_{2}+k_{3}}{K^{4}}+24 \frac{k_{2} k_{3}}{K^{5}}\right) \\
& -i k_{1}^{2} k_{2}^{2} c_{s}\left(\left(k_{1}^{a}\right)^{2}+\left(k_{1}^{b}\right)^{2}\right)\left(\frac{4}{K^{3}}-6 \frac{k_{2}-2 k_{3}}{K^{4}}-24 \frac{k_{2} k_{3}}{K^{5}}\right) \\
& -i k_{1}^{2} k_{2}^{2} c_{s}^{3}\left(\frac{4}{K}-\frac{k_{1}+k_{2}-2 k_{3}}{K^{2}}+2 \frac{k_{1} k_{2}-2 k_{2} k_{3}-2 k_{1} k_{3}}{K^{3}}+6 \frac{k_{1} k_{2} k_{3}}{K^{4}}\right) \\
& +2 i k_{2}^{2} c_{s}\left(\left(k_{1}^{a}\right)^{2}+\left(k_{1}^{b}\right)^{2}\right)\left(\frac{2}{K}-\frac{k_{2}-2 k_{1}-2 k_{3}}{K^{2}}-2 \frac{k_{1} k_{2}+k_{2} k_{3}-2 k_{1} k_{3}}{K^{3}}\right. \\
& \left.\left.+6 \frac{k_{1} k_{2} k_{3}}{K^{4}}\right)-2 i k_{1}^{2} k_{2}^{2} c_{s}^{3}\left(\frac{2}{K}-\frac{k_{2}-2 k_{3}}{K^{2}}+\frac{2 k_{2} k_{3}}{K^{3}}\right)\right] \\
& -\frac{n(n-1)}{2} c_{s} H^{2}\left(\frac{1}{c_{V}^{2}}-1\right) \frac{\dot{\phi}_{0}^{2}}{\phi_{0}^{2}}\left[8 k_{1}^{2} k_{2}^{2} k_{3}^{2} i c_{s}^{3} \frac{1}{K^{3}}\right. \\
& +\left(\left(k_{1}^{a}\right)^{2}+\left(k_{1}^{b}\right)^{2}\right)\left(-3 k_{2}^{2} k_{3}^{2} i c_{s}\left(2 \frac{1}{K^{3}}+6 \frac{k_{1}}{K^{4}}\right)\right. \\
& \left.+\vec{k}_{2} \cdot \vec{k}_{3} \frac{i}{c_{s}}\left(2 \frac{1}{K}+2 \frac{k_{1} k_{2}+k_{2} k_{3}+k_{3} k_{1}}{K^{3}}+6 \frac{k_{1} k_{2} k_{3}}{K^{4}}\right)\right) \\
& -2\left(\vec{k}_{1} \cdot \vec{k}_{2}\right) k_{3}^{2} i c_{s}\left(\frac{1}{K}+\frac{k_{1}+k_{2}}{K^{2}}+2 \frac{k_{1} k_{2}}{K^{3}}\right) \\
& \left.+2\left(\left(k_{1}^{a}\right)^{2}\left(k_{2}^{b}\right)^{2}-k_{1}^{a} k_{1}^{b} k_{2}^{a} k_{2}^{b}\right) k_{3}^{2} \frac{i}{c_{s}}\left(2 \frac{1}{K^{3}}+6 \frac{k_{1}+k_{2}}{K^{4}}+24 \frac{k_{1} k_{2}}{K^{5}}\right)\right] \\
& -\frac{4 \sigma c_{s} H^{2}}{M_{p l}^{2} \epsilon}\left[( ( k _ { 1 } ^ { a } ) ^ { 2 } + ( k _ { 1 } ^ { b } ) ^ { 2 } ) \left(24 k_{1}^{2} k_{2}^{2} k_{3}^{2} i c_{s} \frac{1}{K^{5}}\right.\right. \\
& \left.\left.\left.-2 k_{2}^{2} k_{3}^{2} i c_{s}\left(2 \frac{1}{K^{3}}+6 \frac{k_{1}}{K^{4}}\right)+4 k_{1}^{2} k_{2}^{2} k_{3}^{2} i c_{s}^{3} \frac{1}{K^{3}}\right)\right]+ \text { perm. }\right) \text {, }
\end{aligned}
$$

where $a$ and $b$ denote the first and second component of $k$ vector respectively, $K=$ $k_{1}+k_{2}+k_{3}$ and perm. denotes all the other terms obtained by rotating the index 
$(1,2,3)$. All the background value and Hubble constant are calculated at horizon crossing.

\section{References}

[1] A. H. Guth, Phys. Rev. D 23, 347 (1981); A. D. Linde, Phys. Lett. B 108, 389 (1982); A.Albrecht and P. J. Steinhardt, Phys. Rev. Lett. 48, 1220 (1982)

[2] D. N. Spergel et al., "Wilkinson Microwave Anisotropy Probe (WMAP) three year results: implications for cosmology", astro-ph/0603449].

[3] J.Maldacena, "Non-Gaussian features of primordial fluctuations in single field inflationary models," JHEP 0305:013,2003 arXiv:astro-ph/0210603.

[4] V. Acquaviva, N. Bartolo, S. Matarrese and A. Riotto, Second-order cosmological perturbations from inflation, Nucl. Phys. B 667, 119 (2003) arXiv:astro-ph/0209156.

[5] David Seery and James E. Lidsey, "Primordial non-gaussianities in single field inflation" JCAP 0506:003,2005 arXiv:astro-ph/0503692.

[6] David Seery, James E. Lidsey, "Primordial non-Gaussianities from multiple-field inflation", JCAP 0509:011,2005 arXiv:astro-ph/0506056].

[7] X. Chen, M. Huang, S. Kachru and G. Shiu, "Observational Signatures and Non-Gaussianities of General Single Field Inflation," JCAP 0701:002,2007 arXiv:hep-th/0605045.

[8] Daniel Babich, Paolo Creminelli and Matias Zaldarriaga, "The shape of nonGaussianities", JCAP 0408:009,2004 arXiv:astro-ph/0405356].

[9] P. Creminelli, "On non-Gaussianities in single-field inflation," JCAP 0310:003,2003 arXiv:astro-ph/0306122.

[10] N. Arkani-Hameda, P. Creminellia, S. Mukohyamaa and M. Zaldarriagaa, "Ghost Inflation," JCAP 0404:001,2004 arXiv:hep-th/0312100. 
[11] E. Silverstein and D. Tong, "Scalar speed limits and cosmology: Acceleration from D-cceleration," Phys. Rev. D 70:103505,2004 arXiv:hep-th/0310221.

[12] M. Alishahiha, E. Silverstein and D. Tong, "DBI in the Sky," Phys. Rev. D 70:123505, 2004 arXiv:hep-th/0404084].

[13] X. Chen, "Multi-throat brane inflation", Phys. Rev. D 71, 063506 (2005) arXiv:hep-th/0408084.

[14] X. Chen, "Inflation from warped space", JHEP 0508, 045 (2005) arXiv:hep-th/0501184.

[15] S. Kachru, R. Kallosh, A. Linde, J. Maldacena, L. McAllister and S. P. Trivedi, "Towards inflation in string theory", JCAP 0310, 013 (2003) arXiv:hep-th/0308055.

[16] G. R. Dvali and S. H. H. Tye, "Brane inflation", Phys. Lett. B 450, 72 (1999) arXiv:hep-ph/9812483.

[17] G. R. Dvali, Q. Shafi and S. Solganik, "D-brane inflation", arXiv:hep-th/0105203.

[18] C. P. Burgess, M. Majumdar, D. Nolte, F. Quevedo, G. Rajesh and R. J. Zhang, "The inflationary brane-antibrane universe", JHEP 0107, 047 (2001) arXiv:hep-th/0105204.

[19] G. Shiu and S. H. H. Tye, "Some aspects of brane inflation", Phys. Lett. B 516, 421 (2001) arXiv:hep-th/0106274.

[20] J. Garriga and V. F. Mukhanov, "Perturbations in k-inflation," Phys. Lett. B 458, 2191999 arXiv:hep-th/9904176.

[21] C. Armendariz-Picon, T. Damour and V. Mukhanov, "k-inflation," Phys. Lett. B 458, 2091999 arXiv:hep-th/9904075.

[22] A. Connes, "Noncommutative Geometry" (1994).

[23] N. Seiberg, E. Witten, JHEP 9909, 032 (1999) arXiv:hep-th/9908142. 
[24] N. Seiberg, L. Susskind and N. Toumbas, "Strings in background electric field, space/time noncommutativity and a new noncritical string theory", J. High Energy Phys. 06 (2000) 021 arXiv:hep-th/0005040|.

[25] N. Seiberg, L. Susskind and N. Toumbas, "Space/time non-commutativity and causality", J. High Energy Phys. 06 (2000) 044 [arXiv:hep-th/0005015].

[26] J. Gomis and T. Mehen, "Space-time noncommutative field theories and unitarity", Nucl. Phys. B 591 (2000) 265 arXiv:hep-th/0005129.

[27] M. Chaichian, A. Demichev, P. Presnajder, A. Tureanu, "Space-Time Noncommutativity, Discreteness of Time and Unitarity", arXiv:hep-th/0007156.

[28] L. Alvarez-Gaume, J.L.F. Barbon, R. Zwicky, "Remarks on Time-Space Noncommutative Field Theories",JHEP 0105 (2001) 057, arXiv:hep-th/0103069.

[29] C. S. Chu, B. R. Greene and G. Shiu, Mod. Phys. Lett. A16, 2231 (2001) arXiv:hep-th/0011241] .

[30] R. Brandenberger and P. Ho, "Noncommutative space-time, stringy spacetime uncertainty principle, and density fluctuations," Phys.Rev.D66:023517,2002 arXiv:hep-th/0203119.

[31] Q. G. Huang and M. Li, JHEP 0306, 014 (2003) arXiv:hep-th/0304203; Q. G. Huang and M. Li, JCAP 0311, 001 (2003) arXiv:astro-ph/0308458.

[32] S. Tsujikawa, R. Maartens and R. Brandenberger, "Non-commutative inflation and the CMB", Phys. Lett. B 574, 141 (2003) arXiv:astro-ph/0308169.

[33] Dao-jun Liu and Xin-zhou Li, "Non-commutative Power-law Inflation: Mode Equation and Spectra Index", Phys. Lett. B600 (2004) 1-6 arXiv:hep-th/0409075

[34] C.-S. Chu, B.R. Greene and G. Shiu, "Remarks on inflation and noncommutative geometry", Mod. Phys. Lett. A 16 (2001) 2231 arXiv:hep-th/0011241.

[35] R. Easther, B. R. Greene, W. H. Kinney and G. Shiu, "Inflation as a probe of short dis- tance physics", Phys. Rev. D 64, 103502 (2001), arXiv:hep-th/0104102. 
[36] R. Easther, B. R. Greene, W. H. Kinney and G. Shiu, "Imprints of short distance physics on inflationary cosmology", Phys. Rev. D 67, 063508 (2003), arXiv:hep-th/0110226;

[37] A. Mazumdar and M.M. Sheikh-Jabbari, "Noncommutativity in space and primordial magnetic field", Phys. Rev. Lett. 87 (2001) 011301 arXiv:hep-ph/0012363.

[38] F. Lizzi, G. Mangano, G. Miele and M. Peloso, "Cosmological perturbations and short distance physics from noncommutative geometry," JHEP 0206:049,2002 arXiv:hep-th/0203099. 